\title{
Affective Modulation of Working Memory Maintenance: The Role of Positive and Negative Emotions
}

\author{
Ahu Gokce', Artyom Zinchenko², Efsun Annac ${ }^{2}$, Markus Conci and Thomas Geyer \\ Department of Psychology, Kadir Has University, Istanbul, Turkey \\ 2 Department of Psychology, Ludwig-Maximilians-Universität München, Germany
}

ABSTRACT

\section{KEYWORDS}

spatial working memory

retention

IAPS pictures

delayed matching-to-sample task

\begin{abstract}
The present study investigated the impact of task-irrelevant emotional images on the retention of information in spatial working memory (WM). Two experiments employed a delayed matchingto-sample task where participants had to maintain the locations of four briefly presented squares. After a short retention interval, a probe item appeared and participants were required to indicate whether the probe position matched one of the previously occupied square positions. During the retention interval, task-irrelevant negative, positive, or neutral emotional pictures were presented. The results revealed a dissociation between negative and positive affect on the participants' ability to hold spatial locations in WM. While negative affective pictures reduced WM capacity, positive pictures increased WM capacity relative to the neutral images. Moreover, the specific valence and arousal of a given emotional picture was also related to WM performance: While higher valence enhanced WM capacity, higher levels of arousal in turn reduced WM capacity. Together, our findings suggest that emotions up- or down-regulate attention to items in WM and thus modulate the shortterm storage of visual information in memory.
\end{abstract}

\section{INTRODUCTION}

Emotions influence a variety of cognitive functions including attention (Vuilleumier, 2005), cognitive control (Storbeck \& Watson, 2014; Zinchenko et al., 2015), or long-term memory (Seli et al., 2016; Tyng et al., 2017; Zinchenko et al., 2020a). However, the role of affective information during the short-term retention of information in working memory (WM) is still a matter of debate. While there are many studies that show a link between emotional processes and WM, these studies often reveal contradictory findings concerning the facilitatory versus inhibitory effects that emotions play on WM functioning (see, Schweizer et al., 2019, for a recent meta-analysis; Ribeiro et al., 2019, for a review).

It has been suggested that negative emotions impair spatial but not verbal WM in change-detection tasks (Li et al., 2006; Li et al., 2010; for the link between negative emotions and spatial working memory see Storbeck \& Watson, 2014). In contrast, a significant number of other studies did not find any performance detriments arising from negative emotions, but instead reported that negative emotions actually facilitate WM. For instance, Xie and Zhang (2016) presented emotional images prior to a continuous report task that required observers to memorize the color features of a given set of objects. They found that relative to positive and neutral images, negative images lead to more accurate WM representations (see also Öhman et al., 2001 or Spachtholz et al.,

Corresponding author: Ahu Gokce, Department of Psychology, Kadir Has University, Cibali Mah. Hisaralti Cad. No: 17, 34083 Istanbul, Turkey

Email:ahu.gokce@khas.edu.tr 
2014 for comparable findings). However, Xie and Zhang did not find similar effect of negative affective material on WM capacity, that is, an emotion-dependent variation in the number of items that can be retained in WM. Overall, these findings reveal contradictory outcomes on the role of negative emotions on WM representations. While some studies showed emotion-related improvements in WM performance, others showed no effects at all, and other reported emotion-related costs on WM functioning. These overall rather inconsistent results may partly be due to potential confounding factors, such as differences in the arousal versus valence of a given emotional stimulus, the type of WM process investigated (e.g., related to the retention of verbal vs. visuospatial material), or the specific stimulus that is to be memorized (e.g., colors vs. locations of objects; Olivers et al., 2006).

Yet another central feature that might potentially lead to confounds in the above-mentioned studies relates to the fact that the emotional pictures were usually presented prior to the WM task, making it difficult to identify the specific stage or process affected by the emotions (Lavric et al., 2003). Working memory may involve at least three separate processes: encoding, retention, and retrieval of information. Each of these processes could in principle be influenced by emotional content. Affective images presented prior to the task may influence all three WM stages, precluding the specific stage and associated facilitatory or inhibitory mechanisms that are affected by the emotions. For instance, negative emotional stimuli are important environmental inputs (e.g., for the organisms' survival and adaptation), and thus greatly attract attention. Because of this, the encoding of items in WM (which is a prerequisite for appropriate, i.e., goal-directed, actions) may be facilitated by negative affective states. As a result, the capacity of WM for "emotionally-charged" items may increase during the experience of negative affective states (e.g., Spachtholz et al., 2014). However, due to their high attention-grabbing power, negative stimuli may also divert (executive) attention from the currently ongoing (WM) task. As a result, less attentional resources would be available for solving the (WM) task at hand, leading to performance detriments in terms of poorer memory performance (see also Plancher et al., 2019, for a recent demonstration). Thus, when presenting emotional pictures before the to-be-memorized display, the resulting WM performance would always reflect a mixture of facilitatory and inhibitory effects arising from negative emotions on WM encoding versus retrieval. Therefore, the present study attempted to control the influence of emotions on WM performance by presenting the emotional images during the retention period. Such a design allowed to examine whether any affective modulation of WM performance could specifically be associated with the maintenance of information in WM, while at the same time excluding (or minimizing) encoding or retrieval-related influences. An even more fundamental question that could affect the modulation of WM functions by emotions concerns the differences in the overall arousal and/or valence between negative and neutral (control) stimuli. The relative influences of valence and arousal could, for instance, be tested by the addition of a third condition with positive emotional pictures. This would enable to compare WM performance across qualitatively (and, in terms of arousal levels, quantitatively) different emotional (negative and positive) and neutral pictures. This was the approach taken in the present study.

More specifically, we systematically tested any modulatory effects arising from both positive and negative emotional content on WM processing. To this end, we investigated the role emotions play particularly during the retention of spatial information in WM. That is, rather than investigating potential effects of emotions during the encoding of information in WM (or its retrieval), we tested emotion effects after the offset of the memory display during the retention period. Participants were presented with a memory display of four mono-colored items followed by a delay period in which task-irrelevant images (negative and neutral in Experiment 1A; positive and neutral in Experiment 1B) were shown. After the delay period, a probe item was presented and participants had to indicate whether the probe matched one of the sample items or not.

Based on the studies reviewed above, negative emotions could either hamper or facilitate WM retention. In the present study, a disadvantageous emotion effect could be observed because both the processing of negative information and the retention of information in WM require and compete for selective attention and thus hinder the ability to control or represent relevant WM contents (Vogel et al., 2005; Plancher et al., 2019; Mather et al., 2006; Garrison \& Schmeichel, 2019). In contrast, the high behavioral relevance signaled by negative emotional stimuli may also activate higher levels of cognitive control or executive attention. This hypothesis is based on recent work showing that task-irrelevant emotions can enhance attentional control and facilitate performance in a variety of cognitive control tasks, such as the Stroop, flanker, or Simon tasks (e.g., Zinchenko et al., 2017b; for a review, see, e.g., Zinchenko et al., 2020b). If negative emotions increase executive control and thus spare WM capacity storage, then one would expect to find increased number of items stored in WM (e.g., as measured by means of the $K$ capacity estimate; Pashler, 1988). Positive emotions, by contrast, may induce a relatively relaxed, nonattentive (Zinchenko et al., 2020a) or a more flexible mode of processing (Zinchenko et al., 2017a), so that positive images may divert participants' selective attention to a lesser extent, if at all, from the WM task. A related account would assume that the positive images may leave attentional resources almost unaffected (since these images are biologically less relevant; Sakaki et al., 2012), so that WM capacity should be uninfluenced by the positive or neutral emotional content. However, positive emotions may also yield facilitatory effects (Storbeck \& Maswood, 2016; Yang et al., 2013). For instance, positive emotions are often claimed to expand attention and facilitate global perceptual processing (e.g., according to the broaden-and-build hypothesis; Fredrickson, 2004). Assuming that visuospatial material can be represented in WM in an independent, location-specific or in a global, configural fashion (e.g., Gmeindl et al., 2011), it is possible that positive emotions would facilitate the representation of a memory display as an integrated ensemble over singleitem WM representations and thus effectively reduce the burden on WM capacity. 


\section{METHOD}

\section{Participants}

A total of 40 participants from Kadir Has University took part in two Experiments (20 participants each; Experiment 1A: $M_{\text {age }}=22.0, S D$ $=1.21$ years; Experiment 1B: $M_{\text {age }}=20.7, S D=1.92$ years). All participants were enrolled in the psychology undergraduate program and received course credits for their participation. All participants reported normal or corrected-to-normal vision and were naïve about the purpose of the current study. Participants gave written informed consent prior to their participation. The study was approved by the Ethics Committee of Kadir Has University. The experiment was conducted in a single session that lasted around 50 minutes.

Sample size estimation was informed by previous studies on the effects of negative emotions on WM (e.g., Li et al., 2010; King \& Schaefer, 2011; Xie \& Zhang, 2016). On the basis of the number of participants tested and the statistical measures provided by these studies, a sample size of 20 participants was determined to be sufficient for detecting a difference between emotional and neutral images with a power of 0.8 in a single experiment. Thus, on the basis of the studies mentioned above, one would expect WM accuracy to be modulated by negative emotions relative to the neutral baseline, which would be evidenced by a significant emotion main effect. In the current study, we compared two qualitatively different (negative vs. neutral and positive vs. neutral) emotions, and a difference in WM accuracy between the two emotions should be reflected by a 2 -way interaction. In order to test this previously not reported interaction effect involving the between-subjects factor of emotional valence, we doubled our sample size to 40 participants, assuming that positive emotional pictures facilitate WM (see the Introduction section for the references) and that this facilitatory effect is about the same size as the previously reported disadvantageous effects generated by negative emotions, which would require $2 \times 20=40$ participants (cf. Giner-Sorolla, 2018).

\section{Apparatus and Stimuli}

Stimulus presentation and response recording were controlled by an HP ProDesk PC running the Microsoft Windows 10 operating system.
The experiment was programmed by PsychoPy Builder software (version 1.84.2; Peirce, 2007). Participants faced the screen at a viewing distance of approximately $60 \mathrm{~cm}$. Screen resolution was set to $1920 \times$ 1080 pixels. Responses were collected on a standard computer keyboard. The experimental room was dimly lit.

The sample items presented in the memory display consisted of four gray squares (size: $1^{\circ} \times 0.7^{\circ}$ ) presented randomly at four out of eight possible equidistant locations on an invisible circle (diameter: $21^{\circ}$ ). The probe item presented in the test display was identical to the square stimuli presented in memory display. Probes appeared equally likely at each of the eight locations on the invisible circle. In the match condition, the probe appeared at the location of one of the previous, randomly selected memory items. In the nonmatch condition, the probe item was located at a previously empty location in the memory display. There was an equal number of match and nonmatch trials. During the retention phase, that is, after the presentation of the memory display and before the presentation of the probe display, negative and neutral (Experiment 1A) or positive and neutral (Experiment 1B) emotional pictures were presented. The pictures were taken from the International Affective Picture System (IAPS) database (Lang et al., 2008). Eighty pictures with different contents were chosen for each of the negative, neutral, and positive emotion conditions (see Appendix A). For positive and negative pictures, images with relatively high arousal and high valence ratings were chosen in order to maximize the success of the experimental manipulation, that is, to effectively induce the corresponding emotions (mean arousal ratings: positive $=4.88$, negative $=6.04$, neutral $=3.45$; mean valence ratings: positive $=7.54$, negative $=2.49$, neutral $=5.30$. Statistical comparisons revealed that both the arousal and valence ratings differed significantly across negative, neutral and positive picture types (all $p s<.001$ ).

\section{Procedure}

Experiments $1 \mathrm{~A}$ and $1 \mathrm{~B}$ employed a modification of the paradigm introduced by Phillips (1974). The experiments were identical except that different emotional manipulations were presented during the retention phase (negative and neutral pictures in Experiment $1 \mathrm{~A}$ and positive and neutral pictures in Experiment 1B; neutral pictures were identical in both experiments). Prior to each experiment, participants under-

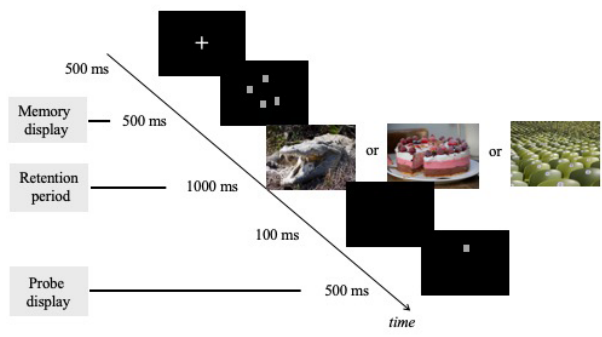

\section{FIGURE 1.}

Example trial sequence. A memory display, consisting of four items, was presented prior to the retention period, during which task-irrelevant negative versus neutral (Experiment 1A) or positive versus neutral (Experiment 1B) pictures were presented. After a brief, blank screen, a single-item probe display appeared. The task was to decide whether the location of the presented probe item matched with any of the four memory items (here: match). Please note that for copyright issues, images other than the actual IAPS images are presented here. The IAPS picture IDs actually used in the experiments are provided in Appendix A. 
went a practice block of 10 trials to become familiar with the task. Each experiment consisted of 10 blocks of 80 trials each, leading to 800 trials in total. Emotional pictures (negative in Experiment 1A, positive in Experiment 1B) were presented in half of the blocks and neutral pictures were presented in the remaining half. In each experiment, five emotional and five neutral blocks were presented in consecutive order. At the beginning of each trial, a white fixation cross was presented on a black background for $500 \mathrm{~ms}$ followed by the presentation of the memory display with four squares located randomly at different positions on a virtual circle. The memory display was shown for $500 \mathrm{~ms}$, followed by a negative, positive, or neutral emotional picture presented for $1000 \mathrm{~ms}$ during the retention interval. To reduce afterimages, a blank screen appeared for another $100 \mathrm{~ms}$ after the emotional picture. Finally, a single square probe item was shown for $500 \mathrm{~ms}$ (see Figure 1). Participants were instructed to respond to the location of the probe stimulus and to decide whether it was the same as or different than any of the four previously shown memory items. Participants pressed the "V" or "N" key for same versus different responses, respectively. Responses had to be executed within $2000 \mathrm{~ms}$ following the onset of the probe item. Error feedback was provided to emphasize accurate responses. After an incorrect response, a warning message ("error") was presented for $500 \mathrm{~ms}$ at the center of the screen.

The use of an independent-group design was motivated by previous studies (e.g., Brunyé et al., 2009) that also tested different groups of participants in different positive versus negative emotion conditions. From a methodological perspective, a between-subject design using blocked (rather than mixed/randomized) presentations of emotional stimuli is also preferable since it reduces undesired variability. Moreover, an important determinant of the quality of an experiment is the number of trials on which the dependent measures are based on (for recent discussions, see Parsons et al., 2019). Given this, we tried to further optimize our experimental paradigm by increasing the number of positive versus negative emotional trials (which, in our view, can be better realized by means of a between-subjects design, which better trades off the number of trials vs. the length of the experiment and thus keeps the burden for participants relatively low). We therefore randomly assigned our participants to two groups: positive versus negative emotions (Experiments $1 \mathrm{~A}$ and $1 \mathrm{~B}$, respectively). In each experiment, positive/negative images were presented in half of the blocks (400 trials), and neutral images were presented in the other half. With this approach, we were able to compare the effects of positive and negative emotional stimuli on WM accuracy (between-group comparison), while also being able to compare positive and negative emotions against a neutral baseline (within-group comparison). Only with the latter neutral image condition, one would be able to assess whether positive versus negative emotions up- or down-modulate the WM performance relative to the neutral baseline.

\section{RESULTS}

Participants' ability to recall item locations was examined by the $K$ parameter: $K=$ set size $\times($ hit rate - false alarm $) /(1$ - false alarm), as introduced by Pashler (1988). The K-parameter was chosen due to its sensitivity and precision in measuring WM capacity (Rouder et al., 2011). In order to examine the valence-specific effects of emotions on spatial WM, we directly compared Experiments $1 \mathrm{~A}$ and $1 \mathrm{~B}$ by means of an omnibus analysis of variance (ANOVA) with the $K$ scores as dependent variable and IAPS picture type (emotional, neutral; withinsubject factor) and emotional valence (negative, positive; betweensubjects factor) as independent variables. It should be noted that the between-subjects factor of emotional valence reflects the comparison across Experiments $1 \mathrm{~A}$ and $1 \mathrm{~B}$ and therefore includes either negative and neutral (Experiment 1A) or positive and neutral (Experiment 1B) picture presentations. Greenhouse-Geisser corrected values are reported in case Mauchly's test of sphericity was significant $(p<.05)$. In cases of significant interactions, post-hoc least significant difference (LSD) tests were used for further comparisons.

The main effect of picture type (emotional vs. neutral) was not significant $(p>.05)$ but there was a reliable main effect of emotional valence (Experiment 1A: negative and neutral images vs. Experiment 1B: positive and neutral images), $F(1,38)=5.21, p<.05, \eta_{\mathrm{p}}{ }^{2}=0.12$. Overall mean $\mathrm{K}$ scores were lower in Experiment $1 \mathrm{~A}(M=1.73, S D=$ 1.21) than in Experiment $1 \mathrm{~B}(M=2.45, S D=0.85)$. Of major theoreti$\mathrm{cal}$ interest was the significant picture type $\times$ emotional valence interaction, $F(1,38)=12.45, p=.001, \eta_{\mathrm{p}}^{2}=0.24$. Post-hoc analyses revealed a significant effect of emotional valence in Experiment 1A (negative vs. neutral pictures), $t(19)=-2.88, p<.01$, Cohen's $d=-0.64$. As illustrated in Figure 2, $K$ scores were smaller for negative as compared to neutral stimuli $(1.59, S D=1.33)$ vs. $1.86, S D=1.11)$. The effect of emotional valence was also significant in Experiment 1B, comparing the effects of positive versus neutral pictures on WM, $t(19)=2.04, p<$ .05 , Cohen's $d=0.45$. WM capacity was higher with positive compared to neutral pictures $(2.52, S D=0.85$ vs. $2.38, S D=0.69)$. Furthermore, a direct comparison of the $K$ scores between negative and positive images was also significant, $t(38)=-2.66, p=.01$, Cohen's $d=-0.84$,

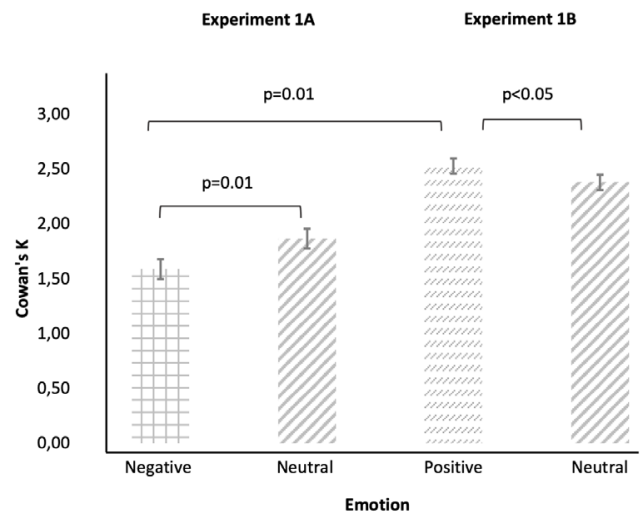

FIGURE 2

The WM capacity estimate K (and associated within-subject error bars) in the spatial delayed match-to-sample task when negative versus neutral (Experiment 1A, left) or positive versus neutral (Experiment 1B, right) emotional IAPS stimuli were presented during the WM retention interval. 
while the difference between the two neutral conditions only revealed borderline significance, $t(38)=-1.76, p=.08$, Cohen's $d=-0.55$ (see Figure 2). The marginal difference between the two identical neutral conditions in the two experiments (see Figure 2) may have resulted from emotional carryover effects. In fact, a comparison of the $K$ scores in the neutral condition in the first block of the experiment (i.e., when participants were not yet presented with other emotional images) showed comparable $K$ scores, $t(38)=1.68, p=.10$, Cohen's $d=0.53$, indicating that performance for the neutral images was comparable in the beginning, while the (marginal) difference only emerged later on when participants were presented with the emotional images. That is, the experience of negative (or positive) pictures may also have impacted processing of the IAPS pictures that were originally rated as neutral (Schmidt \& Schmidt, 2016; Zinchenko et al., 2020a). Such differences can thus be explained by a modulation in emotional quality of the neutral images when accompanied by positive or negative emotional images (Schneider et al., 2016).

In order to test whether WM performance was influenced by response bias, an additional analysis was performed which computed the response bias $C$ derived from the signal detection theory: $C=-0.5 \times$ $\left(z_{\text {hit }}+z_{\text {false alarm }}\right)$ (MacMillan \& Creelman, 2005). Although the above analyses of the hit and false-alarm rates by means of the $K$ memory capacity parameter provide evidence that emotions modulate WM (i.e., $K$ score was highest with positive, intermediate with neutral, and lowest with negative emotional stimuli), it remains possible that emotions also influence the decision-making process, such as a reduction of the threshold required for initiating a response (Kane et al., 2007). This would, for instance, be reflected by a more "liberal" response criterion $C$ for positive relative to neutral (and negative) emotional pictures. However, the comparison of the $C$ parameter across the two experiments revealed neither a main effect of picture type (emotional vs neutral: $\left.F[1,38]=0.01, p>.05, \eta_{\mathrm{p}}{ }^{2}=0.00\right)$ nor of valence (Experiment $1 \mathrm{~A}$ negative: $0.12, S D=0.22$; neutral: $0.11, S D=0.19$; Experiment $1 \mathrm{~B}$ positive: $-0.03, S D=0.20$; neutral: $-0.03, S D=0.50), F(1,38)=2.91, p>.05$, $\eta_{\mathrm{p}}{ }^{2}=0.07$. The interaction between these two factors was also statistically not significant: $F(1,38)=0.04, p>.05, \eta_{\mathrm{p}}{ }^{2}=0.001$. The response criterion $\mathrm{C}$ was thus uninfluenced by the emotional manipulation.

Finally, an additional correlational analysis was carried out in order to establish the specific role of valence and arousal in the presented IAPS images. This was possible because each image in the IAPS database (Lang et al., 2008) is associated with a specific valence and arousal rating. Valence reflects the evoked negative or positive emotion of each image. The mean valence ratings of the selected IAPS pictures were $2.49(S D=0.86)$ and $7.54(S D=0.34)$ for negative and positive images, respectively, while for the neutral images, the mean rating was 5.30 (SD $=0.59)$. The mean arousal ratings were $6.04(S D=0.77), 3.45(S D=$ $0.96)$, and $4.88(S D=0.96)$ for negative, neutral, and positive images, respectively. The image-specific valence and arousal ratings for the presented negative, positive, and neutral pictures were correlated with the overall accuracy (in \%) in the WM task. For this analysis, the data from the two experiments were combined and the WM performance for the neutral images was averaged across Experiments $1 \mathrm{~A}$ and 1B. Of note, the ratings of the IAPS pictures varied continuously and such correlational analyses have been widely used in a variety of previous studies (e.g., Calvo \& Avero, 2009; Grühn \& Scheibe, 2008; MacNamara et al., 2011; Sen et al., 1983; Tok et al., 2010). The results showed that accuracy was positively correlated with valence $(r=0.35, p<.001)$, while it revealed a significant negative correlation with arousal ratings $(r=$ $-0.22, p<.001)$. These correlations thus show that valence and arousal have opposing effects on the performance. An increase in valence leads to an improvement in WM performance, whereas corresponding increase in the arousal conversely results in reduced WM performance.

\section{DISCUSSION}

The present study investigated the role of positive and negative emotions on the retention of information in spatial WM by using a delayed matching-to-sample task. In the experiments, emotional contents were presented during the retention interval during which the previously encoded spatial information had to be maintained until the subsequent retrieval (and comparison to a probe item). Our results show a clear effect of the emotional pictures on WM capacity: negative relative to neutral emotion pictures reduce the WM capacity whereas positive emotional pictures lead to an increase in WM capacity relative to neutral pictures. This overall increase in capacity with positive emotional content of the pictures was also revealed by means of a positive correlation between the individual valence of the presented images and the associated overall memory performance. Conversely, a negative correlation was revealed between the arousal ratings of the presented images and WM performance, indicating that higher arousal levels impair WM performance. Thus the valence and arousal of a given image reveals opposing effects on WM and the variability of these emotional dimensions (or their relative strength) may thus partly explain why previous studies often revealed inconsistent patterns in their results.

It should be noted that the emotional pictures presented during the retention interval were completely task-irrelevant, meaning that participants had no explicit instruction to process these images. This is different from previous approaches (e.g., Plancher et al., 2019), where participants had to explicitly categorize emotional images during the WM task. Overall, this can be taken to suggest that both the explicit processing of emotional information in a picture and the mere exposure to the emotional images (without any associated task) modulate WM capacity.

Our observation of valence-specific effects is in line with several previous studies, though they also significantly extend it. For instance, Li et. al. (2010) found that negative pictures presented prior to a delayed matching-to-sample (spatial) task altered electrophysiological WM correlates, in particular the P3b waveform, which is assumed to reflect encoding and recall from WM (Ruchkin et al., 1990), and is attenuated in response to poorly retained information (Johnson, 1988). Li et al. (2010) found that the P3b was attenuated for negative relative to neutral stimuli. However, this study presented negative images prior to the task, making it difficult to determine which stage is actually influenced by the negative emotions. To overcome this limitation, we presented 
negative and positive images specifically within the retention period (and compared them against neutral pictures), thus being able to test emotional influences specifically at this stage. A potential proposal to account for the effect of emotions during the retention phase relates to the idea of a strong interplay between WM capacity and selective attention, or filtering (Liesefeld et al., 2014). Filtering can be considered as a cognitive capability: The better participants are able to process relevant information, or-as is important for the present task-to shield it from interference during WM maintenance by suppressing task-irrelevant information, the more WM resources are available for the task at hand (Vogel et al., 2005). However, negative emotions may crucially interfere with effective shielding because the potential behavioral relevance of negative emotional contents may impair shielding and thus reduce the availability of WM resources for the task-relevant information (see van den Berg et al., 2014; Oberauer et al., 2016, for different ideas on WM capacity limitations). In this view, negative emotions are particularly strong cues that attract external attention (to sensory events), and as a result, internal attention, the processing and the maintenance of internally generated information, is impaired (see Chun et al., 2011). In a sense, negative emotions could thus be seen as acting like salient (but task-irrelevant) distractors in other visual (search) tasks, capturing attention and delaying detection of response-relevant target information, at least under some conditions (Geyer et al., 2008; Gaspar \& McDonald, 2014). Note that filtering could have been achieved by complementary attention mechanisms that either facilitate relevant information or suppress irrelevant information. Such an explanation in terms of an attentional distraction effect would also be compatible with the finding that socially relevant signals can bias attention during the retention of information in WM (Nie et al., 2018). A performance detriment resulting from the distracting negative emotions is also in line with accounts assuming an interplay between the processing of emotional material and attentional prioritization (i.e., in the dual competition framework; Pessoa, 2009). Taken together, this account considers attention as an executive control capability that is important for overcoming, that is, attenuating, negative emotional states. This may require a thorough sampling of the emotional stimulus presented during the retention phase. However, more careful sampling of negative emotion pictures may then interfere with the efficient retention in the ongoing WM task and lead to reduced performance.

Concerning the facilitatory effects of positive emotion pictures, we suggest an account according to which positive emotions facilitate configural over single item representations and thus enhance the capacity of spatial WM. At the heart of this proposal are the accounts which assume that individual items are stored in WM with regard to their placement within the spatial item configuration (e.g., Jiang et al., 2000; see also Gokce et al., 2013). The amount of information stored in WM can therefore vary depending on the ability to exploit configural cues. The strength of the configural memories might be modulated (increased) by positive emotions, especially because these emotions broaden the focus of attention and the processing of the entire spatial array (Fredrickson, 2004). That is, the representation of the encoded spatial configuration would be enhanced by the exposure to positive emotional images during the retention phase, suggesting that broadening of the attentional focus also occurs when attention is deployed to items maintained in memory, that is, to an already-encoded internal representation (see e.g., Chun et al., 2011; Kiyonaga \& Egner, 2013). Alternatively, facilitatory emotion effects can also be explained by an approach-avoidance motivation (e.g., Elliot \& Thrash, 2002). According to this account, positive images elicit an approach reaction, where participants are more willing to deal with positive stimuli, while negative stimuli, in contrast, lead to an avoidance reaction. Importantly, such an approach-avoidance reaction may also exist for spatial memories, where certain spatial locations can be remembered better if participants approach those locations via the presentation of positive images (e.g., Murty et al., 2011). Of note, the above accounts in terms of the attention filtering/grouping frameworks are only posthoc attempts to coherently explain the current findings. However, they make predictions that can be tested in future research.

A potential limitation of the current study concerns the relationship between the arousal and valence of a given emotional picture. As mentioned above, the negative and positive IAPS images used in this study differed in their respective arousal levels: negative images were somewhat more arousing than positive ones (which is actually a rather common finding in studies that compare effects of positive and negative emotional images, see e.g., Zinchenko et al., 2020a). This difference in arousal levels could also potentially impact the effect of emotions on WM. For instance, the correlational analyses revealed that both arousal and valence affected WM performance, revealing an increase in accuracies with positive valence, but conversely, increased arousal values decreased the overall accuracy. Thus, both valence and arousal influence WM performance, albeit in opposite directions. If arousal values were in turn matched (which is difficult to achieve in practice), we would have expected an even larger difference between the images with positive and negative valence as the matching of arousal levels would eliminate their opposing effects on WM performance. Moreover, these opposing effects of valence and arousal may also explain in part why several previous studies often reported inconsistent results (see also the Introduction section).

To conclude, the present study investigated the impact of taskirrelevant emotional contents presented during the retention of spatial information in WM-one of the most relevant cognitive capabilities needed in everyday life. The results revealed that negative emotions impair while positive emotions facilitate WM performance for visuospatial materials, relative to neutral emotional contents, respectively. These effects were observable specifically during the WM maintenance period, which suggests that emotions in particular influence the ability to hold information in WM. These findings thus help us to broaden our understanding of the interplay between emotional content and WM processing.

\section{REFERENCES}

Brunyé, T. T., Mahoney, C. R., Augustyn, J. S., \& Taylor, H. A. (2009). Emotional state and local versus global spatial memory. Acta Psychologica, 130, 138-146. https://doi.org/10.1016/j.act- 


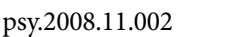

Calvo, M. G., \& Avero, P. (2009). Reaction time normative data for the IAPS as a function of display time, gender, and picture content. Behavior Research Methods, 41, 184-191. https://doi.org/10.3758/ BRM.41.1.184 سلس

Chun, M. M., Golomb, J. D., \& Turk-Browne, N. B. (2011). A taxonomy of external and internal attention. Annual Review of Psychology, 62, 73-101. https://doi.org/10.1146/annurev.psych.093008.100427 المالسلس

Elliot, A. J., \& Thrash, T. M. (2002). Approach-avoidance motivation in personality: approach and avoidance temperaments and goals. Journal of Personality and Social Psychology, 82, 804-818. https:// doi.org/10.1037/0022-3514.82.5.804 السلسلس

Fredrickson, B. L. (2004). The broaden-and-build theory of positive emotions. Philosophical Transactions of the Royal Society of London. Series B: Biological Sciences, 359, 1367-1377. https://doi. org/10.1098/rstb.2004.1512 سلس

Garrison, K. E., \& Schmeichel, B. J. (2019). Effects of emotional content on working memory capacity. Cognition and Emotion, 33, 370-377. https://doi.org/10.1080/02699931.2018.1438989 السلسلس

Gaspar, J. M., \& McDonald, J. J. (2014). Suppression of salient objects prevents distraction in visual search. Journal of Neuroscience, 34, 5658-5666. https://doi.org/10.1523/JNEUROSCI.4161-13.2014 السلسلسل

Geyer, T., Müller, H. J., \& Krummenacher, J. (2008). Expectancies modulate attentional capture by salient color singletons. Vision Research, 48, 1315-1326. https://doi.org/10.1016/j.visres.2008.02.006 السلسلس

Giner-Sorolla R. (2018, January 24). Powering your interaction [Blog post]. https://approachingblog.wordpress.com/2018/01/24/powering-your-interaction-2/

Gmeindl, L., Nelson, J. K., Wiggin, T., \& Reuter-Lorenz, P. A. (2011). Configural representations in spatial working memory: modulation by perceptual segregation and voluntary attention. Attention, Perception, \& Psychophysics, 73, 2130-2142. https://doi.org/10.3758/ s13414-011-0180-0 المالسلس

Gokce, A., Müller, H. J., \& Geyer, T. (2013). Positional priming of popout is nested in visuospatial context. Journal of Vision, 13, 1-16. https://doi.org/10.1167/13.3.32 المالسلس

Grühn, D., \& Scheibe, S. (2008). Age-related differences in valence and arousal ratings of pictures from the International Affective Picture System (IAPS): Do ratings become more extreme with age? Behavior Research Methods, 40, 512-521. https://doi.org/10.3758/ BRM.40.2.512 سلس

Johnson, R. (1988). The amplitude of the P300 component of the event-related potential: Review and synthesis. Advances in Psychophysiology, 3, 69-137. المالسلس

Jiang, Y., Olson, I. R., \& Chun, M. M. (2000). Organization of visual short-term memory. Journal of Experimental Psychology: Learning, Memory, and Cognition, 26, 683-702. https://doi.org/10.1037/02787393.26.3.683 س山سلس

Kane, M. J., Conway, A. R., Miura, T. K., \& Colflesh, G. J. (2007). Working memory, attention control, and the N-back task: a question of construct validity. Journal of Experimental Psychology: Learning, Memory, and Cognition, 33, 615-622. https://doi.org/10.1037/0278-

\subsubsection{5 Ш山س}

King, R., \& Schaefer, A. (2011). The emotional startle effect is disrupted by a concurrent working memory task. Psychophysiology, 48, 269272. https://doi.org/10.1111/j.1469-8986.2010.01062.x المالسلسل

Kiyonaga, A., \& Egner, T. (2013). Working memory as internal attention: Toward an integrative account if internal and external selection processes. Psychonomic Bulletin \& Review, 20, 228-242. https://doi. org/10.3758/s13423-012-0359-y المالسلسل

Lang, P. J., Bradley, M. M., \& Cuthbert, B. N. (2008). International affective picture system (IAPS): Affective ratings of pictures and instruction manual. Technical Report A-8. University of Florida, Gainesville, FL.

Lavric, A., Rippon, G., \& Gray, J. R. (2003). Threat-evoked anxiety disrupts spatial working memory performance: An attentional account. Cognitive Therapy and Research, 27, 489-504. https://doi. org/10.1023/A:1026300619569 الس

Li, X., Chan, R. C. K., \& Luo, Y. (2010). Stage effects of negative emotion on spatial and verbal working memory. BMC Neuroscience, 11: 60. https://doi.org/10.1186/1471-2202-11-60 الس

Li, X., Li, X., \& Luo, Y. J. (2006). Differential influences of negative emotion on spatial and verbal working memory: Evidence from event-related potential and source current density analysis. Neuroreport, 17, 1555-1559. https://doi.org/10.1097/01. wnr.0000234744.50442.2b |لاسلس

Liesefeld, A. M., Liesefeld, H. R., \& Zimmer, H. D. (2014). Intercommunication between prefrontal and posterior brain regions for protecting visual working memory from distractor interference. Psychological Science, 25, 325-333. https://doi. org/10.1177/0956797613501170 السلسلس

Macmillan, N. A., \& Creelman, C. D. (2005). Detection theory: A user's guide (2nd ed.). Mahwah, NJ: Lawrence Erlbaum.

MacNamara, A., Ferri, J., \& Hajcak, G. (2011). Working memory load reduces the late positive potential and this effect is attenuated with increasing anxiety. Cognitive, Affective, \& Behavioral Neuroscience, 11, 321-331. https://doi.org/10.3758/s13415-011-0036-z المالسلس

Mather, M., Mitchell, K. J., Raye, C. L., Novak, D. L., Greene, E. J., \& Johnson, M. K. (2006). Emotional arousal can impair feature binding in working memory. Journal of Cognitive Neuroscience, 18, 614-625. https://doi.org/10.1162/jocn.2006.18.4.614 السلسلس

Murty, V. P., LaBar, K. S., Hamilton, D. A., \& Adcock, R. A. (2011). Is all motivation good for learning? Dissociable influences of approach and avoidance motivation in declarative memory. Learning \& Memory, 18, 712-717. https://doi.org/ 10.1101/lm.023549.111 المالسلس Nie, Q. Y., Ding, X., Chen, J., \& Conci, M. (2018). Social attention directs working memory maintenance. Cognition, 171, 85-94. https:// doi.org/10.1016/j.cognition.2017.10.025 سلس

Oberauer, K., Farrell, S., Jarrold, C., \& Lewandowsky, S. (2016). What limits working memory capacity? Psychological Bulletin, 142, 758799. https://doi.org/10.1037/bul0000046 الم

Olivers, C. N. L., Meijer, F., \& Theeuwes, J. (2006). Feature-based memory-driven attentional capture: Visual working memory content affects visual attention. Journal of Experimental Psychology. Human Perception and Performance, 32, 1243-1265. https://doi. 
org/10.1037/0096-1523.32.5.1243 إلسالس

Öhman, A., Flykt, A., \& Esteves, F. (2001). Emotion drives attention: Detecting the snake in the grass. Journal of Experimental Psychology: General, 130, 466-478. https://doi.org/10.1037/0096-3445.130.3.466 1لس Parsons, S., Kruijt, A. W., \& Fox, E. (2019). Psychological science needs a standard practice of reporting the reliability of cognitive-behavioral measurements. Advances in Methods and Practices in Psychological Science, 2, 378-395. https://doi.org/10.1177/2515245919879695 1لس

Pashler, H. (1988). Familiarity and visual change detection. Attention, Perception \& Psychophysics, 44, 369-378. https://doi.org/10.3758/ BF03210419 الفيلس

Peirce, J. W. (2007). PsychoPy-Psychophysics software in Python. Journal of Neuroscience Methods, 162, 8-13. https://doi.

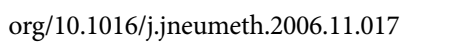

Pessoa, L. (2009). How do emotion and motivation direct executive control? Trends in Cognitive Sciences, 13, 160-166. https://doi. $\operatorname{org} / 10.1016 /$ j.tics.2009.01.006 المالسلس

Phillips, W. A. (1974). On the distinction between sensory storage and short-term visual memory. Perception \& Psychophysics, 16, 283-290. https://doi.org/10.3758/BF03203943 السلسلس

Plancher, G., Massol, S., Dorel, T., \& Chainay, H. (2019). Effect of negative emotional content on attentional maintenance in working memory. Cognition and Emotion, 33, 1489-1496. https://doi.org/10. 1080/02699931.2018.1561420 السلسلس

Ribeiro, F. S., Santos, F. H. D., \& Albuquerque, P. B. (2019). How does allocation of emotional stimuli impact working memory tasks? An overview. Advances in Cognitive Psychology, 15, 155-168. https://doi. org/10.5709/acp-0265-y السلسلس

Rouder, J. N., Morey, R. D., Morey, C. C., \& Cowan, N. (2011). How to measure working memory capacity in the change detection paradigm. Psychonomic Bulletin \& Review, 18, 324-330. https://doi. org/10.3758/s13423-011-0055-3 كلئلس

Ruchkin, D. S., Johnson Jr, R., Canoune, H., \& Ritter, W. (1990). Shortterm memory storage and retention: An event-related brain potential study. Electroencephalography and clinical Neurophysiology, 76, 419-439. https://doi.org/10.1016/0013-4694(90)90096-3 المالسلس

Sakaki, M., Niki, K., \& Mather, M. (2012). Beyond arousal and valence: The importance of the biological versus social relevance of emotional stimuli. Cognitive, Affective, \& Behavioral Neuroscience, 12,

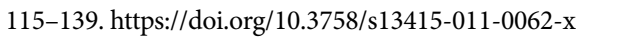

Schmidt, S. R., \& Schmidt, C. R. (2016). The emotional carryover effect in memory for words. Memory, 24, 916-938. https://doi.org/10.1080

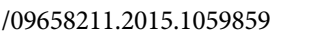

Schneider, I. K., Veenstra, L., van Harreveld, F., Schwarz, N., \& Koole, S. L. (2016). Let's not be indifferent about neutrality: Neutral ratings in the International Affective Picture System (IAPS) mask mixed affective responses. Emotion, 16, 426-430. https://doi.org/10.1037/

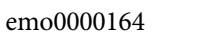

Schweizer, S., Satpute, A. B., Atzil, S., Field, A. P., Hitchcock, C., Black, M., Barrett, L.F., \& Dalgleish, T. (2019). The impact of affective information on working memory: A pair of meta-analytic reviews of behavioral and neuroimaging evidence. Psychological Bulletin, 145,
566-609. http://dx.doi.org/10.1037/bul0000193 سلس

Seli, P., Wammes, J. D., Risko, E. F., \& Smilek, D. (2016). On the relation between motivation and retention in educational contexts: The role of intentional and unintentional mind wandering. Psychonomic Bulletin and Review, 23, 1280-1287. https://doi.org/10.3758/s13423-

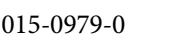

Sen, A., Jensen, A. R., Sen, A. K., \& Arora, I. (1983). Correlation between reaction time and intelligence in psychometrically similar groups in America and India. Applied Research in Mental Retardation, 4,

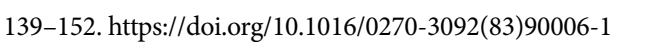

Spachtholz, P., Kuhbandner, C., \& Pekrun, R. (2014). Negative affect improves the quality of memories: Trading capacity for precision in sensory and working memory. Journal of Experimental Psychology: General, 143, 1450-1456. https://doi.org/10.1037/xge0000012 السلسلسلكا

Storbeck, J., \& Maswood, R. (2016). Happiness increases verbal and spatial working memory capacity where sadness does not: Emotion, working memory and executive control. Cognition and Emotion, 30, 925-938. https://doi.org/10.1080/02699931.2015.1034091 السلسلس

Storbeck, J., \& Watson, P. (2014). Verbal makes it positive, spatial makes it negative: Working memory biases judgments, attention, and moods. Emotion, 14, 1072-1086. https://doi.org/10.1037/ a0037327 الفيلس

Tok, S., Koyuncu, M., Dural, S., \& Catikkas, F. (2010). Evaluation of International Affective Picture System (IAPS) ratings in an athlete population and its relations to personality. Personality and Individual

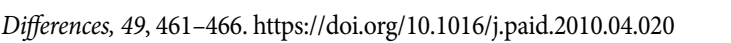

Tyng, C. M., Amin, H. U., Saad, M. N., \& Malik, A. S. (2017). The influences of emotion on learning and memory. Frontiers in Psychology, 8:1454. https://doi.org/10.3389/fpsyg.2017.01454 سلس

van den Berg, R., Awh, E., \& Ma, W. J. (2014). Factorial comparison of working memory models. Psychological Review, 121, 124-149. https://doi.org/10.1037/a0035234 المالسلس

Vogel, E. K., McCollough, A. W., \& Machizawa, M. G. (2005). Neural measures reveal individual differences in controlling access to working memory. Nature, 438, 500-503. https:/doi.org/10.1038/ nature 04171 الس الس

Vuilleumier, P. (2005). How brains beware: neural mechanisms of emotional attention. Trends in Cognitive Sciences, 9, 585-594. https://doi. org/10.1016/j.tics.2005.10.011 الفلسلسلس

Xie, W., \& Zhang, W. (2016). Negative emotion boosts quality of visual working memory representation. Emotion, 16, 760-774. https://doi. org/10.1037/emo0000159 الس السلس

Yang, H., Yang, S., \& Isen, A. M. (2013). Positive affect improves working memory: Implications for controlled cognitive processing. Cognition \& Emotion, 27, 474-482. https://doi.org/10.1080/026999 31.2012.713325 السلس

Zinchenko, A., Kanske, P., Obermeier, C., Schröger, E., \& Kotz, S. A. (2015). Emotion and goal-directed behavior: ERP evidence on cognitive and emotional conflict. Social Cognitive and Affective Neuroscience, 10, 1577-1587. https://doi.org/10.1093/scan/nsv050 المسلسلس

Zinchenko, A., Obermeier, C., Kanske, P., Schröger, E., \& Kotz, S. A. (2017a). Positive emotion impedes emotional but not cognitive con- 
flict processing. Cognitive, Affective, \& Behavioral Neuroscience, 17, 665-677. https://doi.org/10.3758/s13415-017-0504-1 السلسلسلا

Zinchenko, A., Obermeier, C., Kanske, P., Schröger, E., Villringer, A., \& Kotz, S. A. (2017b). The influence of negative emotion on cognitive and emotional control remains intact in aging. Frontiers in Aging

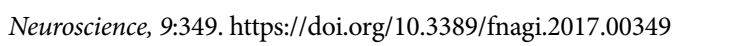

Zinchenko, A., Geyer, T., Müller, H.J., \& Conci, M. (2020a). Affective modulation of memory-based guidance in visual search: Dissociative role of positive and negative emotions. Emotion, 20, 1301-1305. https://doi.org/10.1037/emo0000602 السلبل
Zinchenko, A., Kotz, S. A., Schröger, E., \& Kanske, P. (2020b). Moving towards dynamics: Emotional modulation of cognitive and emotional control. International Journal of Psychophysiology, 147, 193201. https://doi.org/10.1016/j.ijpsycho.2019.10.018

RECEIVED 19.05.2020 | ACCEPTED 13.01.2021 


\section{APPENDIX A: IAPS PICTURES (IDS) USED IN THE PRE- SENT STUDY}

Positive: $1340,1440,1460,1500,1540,1590,1600,1604,1610,1620$, 1630, 1710, 1721, 1750, 1811, 1920, 1999, 2035, 2040, 2045, 2058, 2070, 2080, 2091, 2158, 2222, 2274,2311, 2340, 2345, 2387, 2388, 2398, 2530, 2550, 2650,4614, 4640,4641, 5010, 5200, 5202, 5470, $5480,5600,5621,5623,5660,5760,5780,5781,5811,5820,5830$, 5831, 5833, 5910, 5982, 7200, 7230, 7260, 7280, 7330, 7580, 8030, $8080,8163,8180,8190,8200,8350,8370,8380,8420,8461,8490$, $8497,8499,8501,8540$.

Negative: 1052, 1111, 1113, 1120, 1200, 1205, 1220, 1274, 1280, 1300, 2053, 2352.2, 2661, 2691, 2730, 2750, 2800, 2981, 3000, 3010, 3015, 3030, 3051, 3060, 3061, 3064, 3068, 3069, 3071, 3080, 3100, 3110, $3120,3130,3140,3150,3160,3181,3250,3301,3400,3500,3530$, $3550,6020,6190,6211,6212,6312,6313,6350,6360,6540,6550$, $6560,6570,6821,6840,8480,9040,9042,9050,9140,9252,9253$, 9290, 9301, 9400, 9410, 9415, 9420, 9421, 9430, 9433, 9520, 9560, 9561, 9570, 9571, 9900 .
Neutral: 2880, 5510, 5891, 7001, 7004, 7017, 7025, 7038, 7039, 7042, $7043,7050,7053,7056,7059,7060,7077,7140,7150,7160,7161$, $7165,7170,7175,7179,7185,7186,7187,7188,7190,7192,7207$, $7211,7217,7233,7249,7354,7484,7495,7496,7497,7500,7510$, $7512,7513,7700,7705,7900,8162,8341,7030,7040,7052,7130$, 7061, 7620, 8211, 8465, 7640, 7950, 8250, 7034, 7041, 7080, 7137, $7234,7503,7545,8260,7006,7035,7055,7090,7235,7490,7504$, $7547,8280,7009,7036$. 